\title{
Desenvolvimento e validação de método para extração e quantificação através de HPLC com índice de refração para lactose em leite pasteurizado
}

\author{
Vinicius Ricardo Acquaro Jr., Tiago Bervelieri Madeira, Drielle Caroline Castilho, \\ Lycio Shinji Watanabe, Yuri Renan Bovolenta, Suzana Lucy Nixdorf* \\ Laboratório DIA, Departamento de Química, Centro de Ciências Exatas, Universidade Estadual de Londrina - UEL, \\ Jardim Portal de Versalhes 1, Campus Universitário, CP 10011, Cep 86057970, Londrina, PR, Brasil \\ e-mail: snixdorf@uel.br
}

Resumo

Um método simples, preciso, exato, linear em ampla faixa de trabalho e baseado em princípios da Química Verde foi desenvolvido e validado para a extração e determinação da lactose utilizando cromatografia líquida com deteç̧ão por índice de refração. A lactose, no teor mínimo definido pelo MAPA (Ministério da Agricultura, Pecuária e Abastecimento), foi o marcador de qualidade escolhido, por definir o preço do leite e auxiliar na detecção de fraudes. O leite como uma matriz de composição complexa, rica em proteínas, lipídios, carboidratos, sais minerais e vitaminas, exige um pré-tratamento adequado da amostra. Este trabalho visou desenvolver e validar uma metodologia que estabelecesse os parâmetros e condições analíticas, objetivando maximizar a porcentagem de lactose extraída do leite. Para isso, um planejamento estatístico do tipo composto central foi aplicado, considerando o tempo de centrifugação, a intensidade de rotação e a proporção de diluição entre solvente/amostra como variáveis independentes. Os tratamentos estatísticos permitiram determinar a condição de extração otimizada, com taxa de recuperação média de $105 \%$, onde a proporção de etanol: leite foi de (4: 1, v/v), com rotação de $10.000 \mathrm{rpm}$ por 37 minutos. O método proposto apresenta resolução cromatográfica adequada entre a lactulose e a lactose, emprega somente água como fase móvel e etanol no preparo da amostra, em substituição a reagentes dispendiosos e solventes tóxicos. Seu uso representa uma alternativa viável e diferenciada para a determinação do teor de lactose no leite pasteurizado comercial.

Palavras-chave

Carboidrato; etanol; lactose; planejamento experimental; adulteração.

\section{Development and validation of method for extraction and quantification by HPLC with refractive index to lactose in pasteurized milk}

\section{Abstract}

A simple, precise and accurate method was developed and validated to extract and determine lactose over a wide linear working range using liquid chromatography with refractive index detection based on the principles of green chemistry. With its minimum level established by the MAPA (Ministry of Agriculture, 
Livestock and Food Supply of Brazil), lactose has been chosen as a quality marker to set milk prices and facilitate fraud detection. Since milk is a complex matrix rich in proteins, lipids, carbohydrates, mineral salts and vitamins, it requires proper sample pretreatment. The present work was aimed at developing and validating a methodology for establishing parameters and analytical conditions in order to increase lactose percentage extracted from milk. For this purpose, a central composite statistical design with three independent parameters (centrifugation time, rotation intensity and solvent/sample dilution ratio) was employed. The statistical treatments allowed finding optimum extraction conditions - average recovery rate $105 \%$, ethanol: milk ratio $4: 1(\mathrm{v} / \mathrm{v})$, and rotation $10,000 \mathrm{rpm}$ for $37 \mathrm{~min}$. The proposed method with appropriate chromatographic resolution between lactulose and lactose, using water as the mobile phase, and only ethanol for sample pretreatment, instead of expensive reagents and toxic solvents, is a distinct and feasible alternative to determine lactose contents of pasteurized milk.

Keywords

Carbohydrates; ethanol; lactose; experimental design; adulteration.

\section{Introdução}

O Brasil é um dos maiores produtores mundiais de leite, detendo a quarta posição, atrás dos Estados Unidos, Índia e China. O leite, dentre as commodities agropecuárias, vem depois da soja, cana-de-açúcar e milho. A sua produção em 2011 alcançou 32,1 bilhões de litros, frente aos 27,6 bilhões ofertados em 2008, representando uma taxa de crescimento anual de 5,3\%. Essa produtividade tem impulsionado a elevação do consumo, que está em 173 litros de leite per capita por ano ${ }^{[1]}$.

O leite é considerado o mais nobre dos alimentos, como uma importante fonte nutritiva para o ser humano, devido a sua composição rica em proteínas, gorduras, carboidratos, sais minerais e vitaminas ${ }^{[2,3]}$. Essa composição pode variar em função da fisiologia, raça, estado de saúde e alimentação dos animais ${ }^{[3-5]}$. Todavia, nos últimos anos têm-se verificado alterações por constantes adulterações, com adição de água ou neutralizantes ou substâncias que ameaçam a segurança alimentar como: melaminas, ureia, nitrato de amônia, urina e peróxido de hidrogênio $^{[4,6]}$.
O Ministério da Agricultura Pecuária e Abastecimento (MAPA), visando dar um norte ao setor quanto à qualidade, determina valores mínimos em termos de variação dos componentes do leite. Para a gordura e a lactose são exigidos teores mínimos de 3,0\% e 4,3\%, respectivamente. Para o extrato seco total é requerido um mínimo de $11,5 \%$, enquanto para o extrato seco desengordurado o teor mínimo é de $8,5 \%$, com quantidade mínima de proteína de $3,0 \%{ }^{4}$.

Nesse sentido, determinar o teor de lactose, o carboidrato mais abundante no leite, é importante, uma vez que esse açúcar contribui para as propriedades funcionais e sensoriais do produto; agrega valor econômico, já que o preço do leite baseia-se no teor de sólidos; e é um dos parâmetros de qualidade, que pode auxiliar na detecção das fraudes. Portanto, é fundamental para a saúde pública que estudos relacionados a métodos capazes de comprovar a autenticidade do leite e de detectar adulterações pela incorporação de substâncias desconhecidas sejam desen$\operatorname{volvidos}^{[7]}$.

O método oficial para a determinação de lactose em leite, o 984.15 da AOAC (The Association of Analytical Communities) é 
complexo e demorado, requerendo o preparo semanal de dois dos sete reagentes utilizados. Envolve a hidrólise enzimática da lactose em glicose e galactose em $\mathrm{pH}$ 6,6 pela $\beta$-galactosidase, com subsequente oxidação da $\beta$-galactose, liberando o ácido galacturónico em $\mathrm{pH}$ 8,6, e redução do dinucleótido de nicotinamida-adenina $(\mathrm{NAD}+)$, catalisado pela $\beta$-galactose desidrogenase. A concentração da lactose é calculada pela quantidade formada do NAD reduzido medida a $340 \mathrm{~nm}^{[8]}$.

Um método mais simples, rápido e preciso empregando HPLC é descrito na literatura ${ }^{[8]}$, porém utiliza o detector específico Corona charged aerosol detector (CAD Thermo Scientific Dionex, EUA), o detector eletroquímico com amperometria pulsada ${ }^{[9]}$ e de espalhamento de luz evaporativo ${ }^{[10]}$, equipamento nem sempre disponível nos laboratórios de controle de qualidade. Outra possibilidade apontada está em quantificar a lactose por cromatografia líquida de alta eficiência com índice de refração (HPLCRID) $)^{[11]}$.

Entretanto, a complexidade da matriz do leite exige que se realize um clean-up na fase do preparo da amostra, visando evitar danos à coluna cromatográfica. Diferentemente da literatura, ao invés de se utilizar de 70\% a 75\% de acetonitrila na fase móvel ${ }^{[11,12]}$, neste estudo utiliza-se somente água na fase móvel. Outra diferença é que se realiza a desproteinização etanólica em substituição à precipitação, utilizando-se ácido acético, acetato de zinco e ferrocianeto de potássio, acetato de chumbo, oxalato de sódio e hidrogeno fosfato de sódio ${ }^{[11]}$, ou ela é feita com o reativo de Carrez ${ }^{[12]}$. O etanol foi selecionado como pré-tratamento organossolvente por: desnaturar as proteínas alterando o grau de hidratação no soro do leite, ser um reagente de fácil obten- ção, relativamente de baixo custo, e apresentar baixa toxicidade, preceitos fundamentais da Química Verde ${ }^{[8,10]}$.

O objetivo do presente estudo foi maximizar a extração da lactose, seguindo os preceitos da química limpa e planejamento fatorial, utilizando o etanol como solvente no preparo das amostras e empregando a água como fase móvel na cromatografia líquida de alta eficiência a fim de determinar seu teor no leite pasteurizado.

\section{Materiais e métodos}

\subsection{Materiais}

Para as análises, utilizaram-se: um padrão de lactose obtido da Sigma-Aldrich (St. Louis, MO, EUA); etanol grau p.a. (Synth, Brasil); e água ultrapura obtida através de um sistema Milli-Q ${ }^{\circledR}$ (Millipore, Billerica, MA, EUA). As amostras foram de leite pasteurizado, fornecidas pelo centro mesorregional de excelência em tecnologia do leite do Norte Central da Universidade Estadual de Londrina, Paraná, Brasil.

\subsection{Equipamentos}

$\mathrm{Na}$ centrifugação, empregou-se uma ultracentrífuga (Hitachi, Tóquio, Japão) CP 70MX com rotor P70AT, de velocidade máxima de $70.000 \mathrm{rpm}$, pertencente ao Laboratório Multiusuário LAPA da UEL.

A determinação da lactose foi feita no sistema cromatográfico a líquido de alta eficiência (Shimadzu, Quioto, Japão) composto por bomba LC-10A, com sistema gerenciador de gradiente e desgaseificador, e detector por índice de refração RID-10A, gerenciados pelo software Class-VP. Utilizou-se uma válvula injetora Rheodyne 7125 com alça de amostragem de 20,0 $\mu \mathrm{L}$ e uma coluna de troca catiônica de Pb (II) - Aminex HPX-87P 
(300 mm $\times 7,8 \mathrm{~mm}, 9,0 \mu \mathrm{m}$, Biorad, EUA), mantida a $80{ }^{\circ} \mathrm{C}$. A vazão da água, empregada como fase móvel, foi de $0,8 \mathrm{~mL} \mathrm{~min}^{-1}$.

\subsection{Planejamento estatístico}

Avaliou-se o efeito do tempo de centrifugação, da intensidade da rotação da centrífuga e da proporção de diluição (solvente: amostra, v/v), seguindo um planejamento estatístico composto central, com três variáveis independentes e cinco níveis de variação, conforme Tabela 1. Foram realizados 16 experimentos: oito nos pontos fatoriais $(-1,+1)$; seis nos pontos axiais $(-1,68$, $+1,68)$; e dois no ponto central $(0,0)$, inteiramente aleatorizados.

\subsection{Extração}

Para a extração partiu-se de cinco mililitros de leite pasteurizado, adicionando-se o solvente (etanol) em diferentes proporções de diluição (Tabela 1), e agitando-se manualmente por cerca de um minuto. O tubo foi levado à ultracentrífuga (CP 70MX) e submetido a diferentes tempos e rotações (Tabela 1). O sobrenadante foi passado por papel filtro qualitativo e por cartucho SepPak $^{\circledast}$ Vac 3cc (500 mg) C18 (Waters $\left.{ }^{\circledast}\right)$. Após a filtração, a amostra foi levada à evaporação completa no speed vacuum (Thermo Jouan RC10-22). Em seguida, foi ressuspendida em 10 mililitros de água ultrapura e analisada por cromatografia líquida de alta eficiência com detector de índice de refração (HPLC-RID).

\subsection{Validação}

A metodologia foi validada avaliando-se a seletividade, precisão (repetitividade e intermediária), linearidade, faixa dinâmica de trabalho, limite de detecção e de quantificação e a exatidão pelas taxas de recuperação.

\section{Resultados e discussão}

A validação da metodologia foi realizada de acordo com parâmetros e critérios de procedimentos da International Conference on Harmonization $^{[13]}$.

\subsection{Seletividade}

A seletividade foi comprovada pelo tempo de retenção da lactose idêntico para o padrão externo e para as amostras e pelo aumento proporcional das áreas dos picos, quando efetuadas as adições de padrão. A ausência de resposta analítica, quando injetada água ultrapura no tempo de retenção da lactose, comprova que não houve interferentes ${ }^{[13]}$.

\subsection{Precisão (repetitividade)}

A precisão foi determinada pela repetitividade de 10 injeções sucessivas do padrão analítico $^{[14]}$ de $65,0 \mathrm{~g} \mathrm{~L}^{-1}$ de lactose, obtendo-se um desvio padrão de $\pm 0,03$ e desvio padrão relativo (DPR) de $0,80 \%$, já para o tempo de retenção o desvio padrão foi de $\pm 0,04$ e o DPR, de $0,51 \%$.

Tabela 1 Variáveis independentes e níveis de variação do planejamento fatorial composto central.

\begin{tabular}{lccccc}
\hline \multirow{2}{*}{ Variáveis independentes } & \multicolumn{5}{c}{ Níveis de variação } \\
\cline { 2 - 6 } & $-1,68$ & -1 & 0 & +1 & $+1,68$ \\
\hline$X_{1}=$ Tempo de rotação $(\mathrm{min})$ & 3,12 & 10 & 20 & 30 & 36,80 \\
$X_{2}=$ Rotação $(\mathrm{rpm})$ & 3.200 & 5.000 & 7.500 & 10.000 & 11.700 \\
$X_{3}=$ Proporção da diluição $(\mathrm{mL})$ & $0,32: 1$ & $1: 1$ & $2: 1$ & $3: 1$ & $3,68: 1$ \\
\hline
\end{tabular}




\subsection{Linearidade e faixa dinâmica de trabalho}

As três curvas analíticas foram levantadas injetando-se aleatoriamente em três dias distintos a triplicata das concentrações dos padrões de lactose a 10,$0 ; 20,0 ; 30,0 ; 40,0 ; 50,0$ e $65,0 \mathrm{~g} \mathrm{~L}^{-1}$, escolhidas de forma a cobrir uma faixa de $50 \%$ a $150 \%$ do valor que se espera encontrar na amostra de estudo ${ }^{[15]}$. Essas curvas foram utilizadas para verificação da: linearidade; faixa dinâmica de trabalho; limites de detecção e quantificação; e precisão intermediária. O coeficiente de correlação (r) estabelecido para os seis pontos da triplicata das três curvas foi de 1,000, considerando-se essa a faixa dinâmica de trabalho, que foi de 10,0-65,0 $\mathrm{g} \mathrm{L}^{-1}$ (Figura 1a).

\subsection{Limite de detecção e de quantificação}

O limite de detecção (LD) foi definido como a concentração do analito que produziu um sinal igual a $3 s$ e o limite de quantificação (LQ), a concentração do analito que produziu um sinal igual a 10 s, onde $s$ é o desvio padrão do ruído medido empregando-se a água como o branco ${ }^{[13]}$. Para o método proposto, o LD para a lactose foi de $0,05 \mathrm{~g} \mathrm{~L}^{-1}$ e o LQ, de $0,15 \mathrm{~g} \mathrm{~L}^{-1}$.

\subsection{Precisão intermediária}

A precisão intermediária do método proposto para a lactose foi considerada adequada, uma vez que não houve desvios nas áreas dos padrões utilizados, o que pode ser observado pela Figura 1a para as três curvas de calibração feitas em três dias distintos e pelo gráfico dos resíduos (Figura 1b), que não apresenta tendências pela distribuição equitativa com baixa dispersão. Já a precisão intermediária calculada a partir dos desvios padrão relativos de uma amostra extraída e analisada em triplicata, em três dias diferentes, por três analistas distintos foi de 9,5\%. Portanto, o método foi considerado preciso, uma vez que os coeficientes de variação foram inferiores a $15 \%$, valor adotado como referência de acordo com a resolução da ANVISA ${ }^{[16]}$.

\subsection{Taxas de recuperação}

O método de extração e determinação proposto demostrou-se exato, uma vez que as taxas de recuperação para a adição de padrão em con-

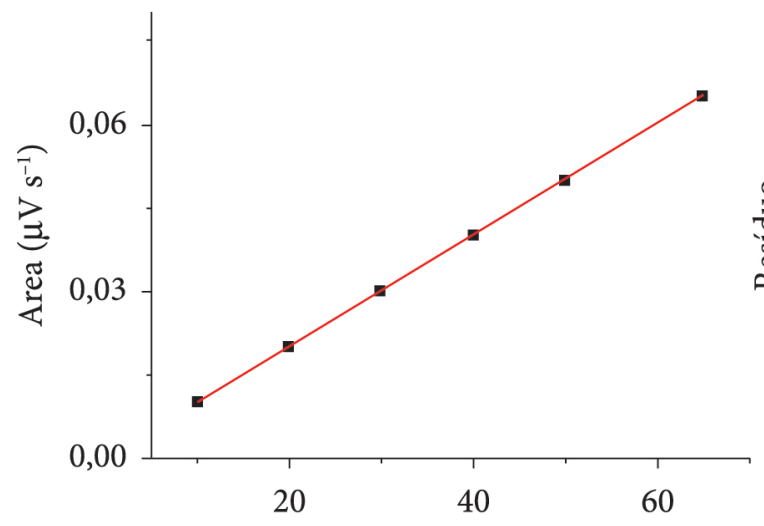

[Lactose] ( $\left.\mathrm{g} \mathrm{L}^{-1}\right)$

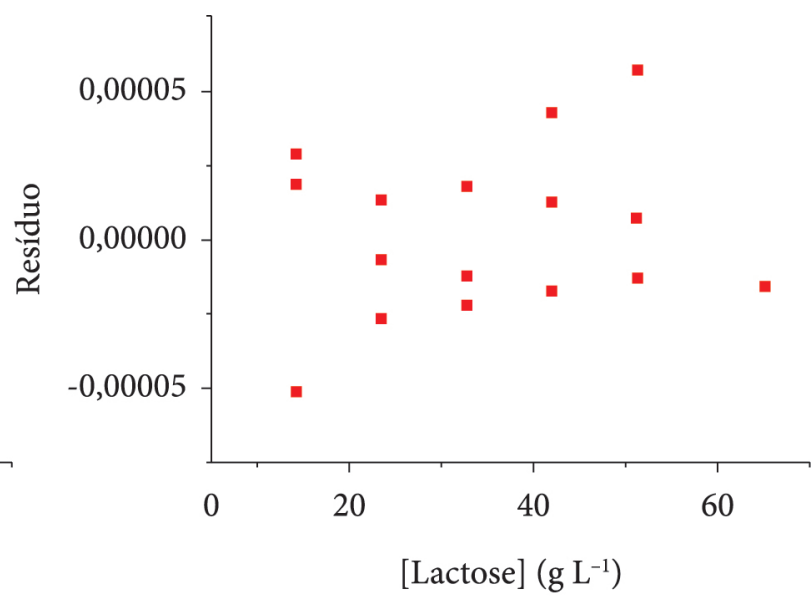

(b)

Figura 1 Curva de calibração para os padrões de lactose de 10,0 $\mathrm{g} \mathrm{L}^{-1}$ a $65,0 \mathrm{~g} \mathrm{~L}^{-1}(\mathrm{~N}=3)$ em três dias distintos (a); gráfico de resíduos (b). 
centrações baixa $\left(1,0 \mathrm{~g} \mathrm{~L}^{-1}\right)$, média $\left(4,3 \mathrm{~g} \mathrm{~L}^{-1}\right) \mathrm{e}$ alta $\left(6,5 \mathrm{~g} \mathrm{~L}^{-1}\right)$ foram de $104,1 \%, 104,6 \%$ e $106,3 \%$, respectivamente. Esses valores estão dentro do intervalo de $70 \%$ a $120 \%$, aceito pela ANVISA ${ }^{[16]}$, com desvios padrão médios de $1,1 \%, 1,0 \%$ e $0,35 \%$, nas triplicatas, respectivamente.

\subsection{Planejamento fatorial}

Pela análise de variância (ANOVA), o único efeito significativo em nível de 5\% ( $\mathrm{p}<0,05)$ foi de $X_{3}$ (Tabela 2), confirmado pelas superfícies de resposta para as três variáveis (Figura 2), que demostram o efeito linear significativo sem curvatura somente para a proporção da diluição (Figura 2b).

As variáveis independentes foram analisadas visando-se obter uma equação capaz de predizer a resposta dentro de um dado intervalo.
Assim, somente as variáveis de maior influência foram conservadas no modelo de regressão para a extração de lactose $(Y)$, dado pela Equação 1:

$Y=-2,759+0,0739 X_{1}-0,133 X_{2}^{2}+0,4618 X_{3}+0,1165 X_{1} X_{2}$

Os valores experimentais de lactose em função dos calculados pelo modelo preditivo (Equação 1) apresentaram um bom ajuste ${ }^{[17]}$, com coeficiente de determinação $\left(R^{2}\right)$ de 0,8304 , explicando $83,04 \%$ da variância dos dados (Figura 3).

A análise da desejabilidade indicou os pontos ótimos de extração como sendo: tempo $(+1,68)$, rotação $(+1)$ e diluição $(+1,68)$. O valor teórico predito pela Equação 1 foi de 3,72\% $(\mathrm{m} / \mathrm{m})$ de lactose na amostra do leite pasteurizado, enquanto o valor experimental foi de $3,74 \%(\mathrm{~m} / \mathrm{m})$.

Tabela 2 Análise de variância dos parâmetros de regressão (ANOVA).

\begin{tabular}{lccccc}
\hline \multicolumn{1}{c}{ Fatores } & SS & $d f$ & $M S$ & $F$ & $p$ \\
\hline Tempo (L) & 0,07467 & 1 & 0,0746 & 1,2126 & 0,294332 \\
Rotação (Q) & 0,21937 & 1 & 0,2193 & 3,5624 & 0,085753 \\
Dilvição (L) & 2,91300 & 1 & 2,9130 & 47,305 & 0,000027 \\
Tempo X Rotação & 0,10850 & 1 & 0,1085 & 1,7621 & 0,211260 \\
Erro & 0,67737 & 11 & 0,0615 & & \\
Total SS & 3,99292 & 15 & & & \\
\hline
\end{tabular}

*(L): Efeito linear; (Q): Efeito quadrático; SS: Soma dos quadrados; df: Graus de liberdade; MS: Quadrado da média; F: Valor calculado; p: Nível de significância.
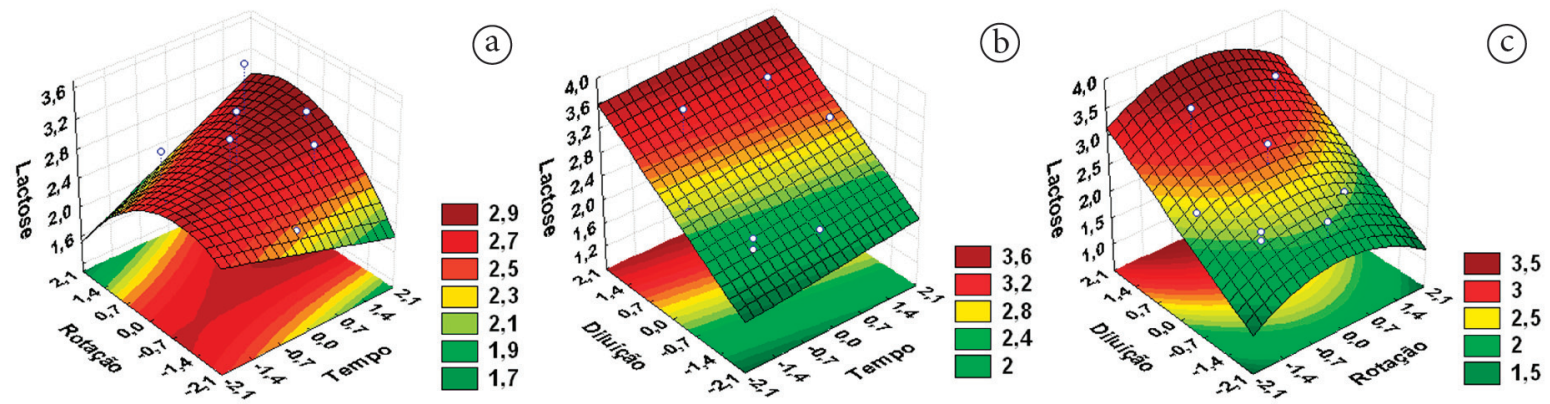

Figura 2 Superfícies de resposta para os efeitos de rotação $X$ tempo (a), diluição $X$ tempo (b) e diluição $X$ rotação (c). 
Considerando-se o efeito linear significativo para a diluição (Figura 2 b) com ausência de máximo, novos testes em relação ao efeito de quantidade de solvente foram realizados com diferentes diluições (4: 1, 4,5: 1 e 5: 1 de etanol: leite, v/v), fixando-se a rotação e o tempo nas condições ótimas da análise de desejabilidade. Os valores de lactose encontrados foram de $4,12 \%(\mathrm{~m} / \mathrm{m}), 4,02 \%(\mathrm{~m} / \mathrm{m})$ e $3,71 \%(\mathrm{~m} / \mathrm{m})$, respectivamente. Nota-se um abaixamento na porcentagem de lactose, atribuída à saturação, já que a solubilidade da lactose em etanol $80 \%$ a $25^{\circ} \mathrm{C}$ é de $0,054 \mathrm{~g} / 100 \mathrm{~g}$ de solvente ${ }^{[18]}$.

A lactose apresenta alta estabilidade no leite, não sofrendo degradação em condições normais ( $\mathrm{pH}$ 6,6-6,8) ou em consequência da mastite, quando a inflamação das glândulas mamárias

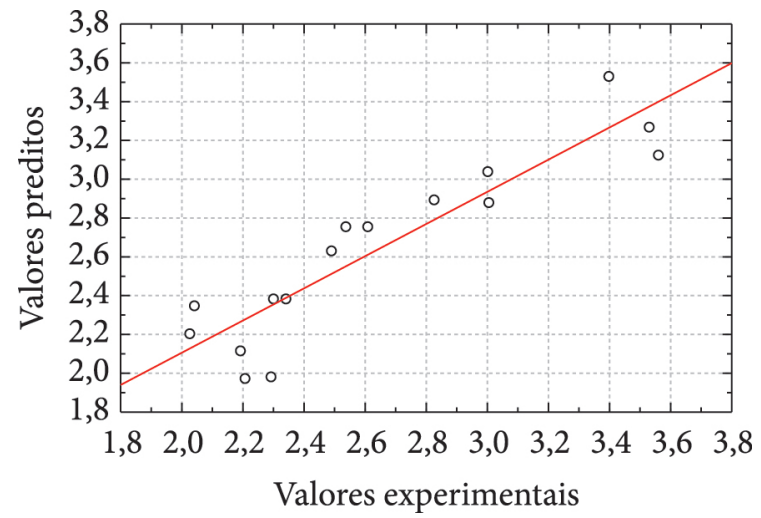

Figura 3 Correlação dos valores experimentais com os previstos pelo modelo. do animal o torna mais alcalino $(\mathrm{pH} 7,3-7,5)^{[19]}$. A presença de microrganismos e a utilização de elevadas temperaturas no processo ${ }^{[10]}$ também não são capazes de converter a lactose em seus monossacarídeos - galactose e glicose, hidrólise essa que ocorre somente no trato intestinal ${ }^{[20,21]}$, pela ação da enzima lactase ${ }^{[10]}$. Por outro lado, durante severos tratamentos térmicos ocorre a produção de um epímero - a lactulose (4-O- $\beta$-dgalactopiranosil-d-frutose ${ }^{[22]}$, formada a partir da lactose (4-O- $\beta$-d-galactopiranosil-d-glicose) pelo rearranjo de Lobry de Bruyn-Alberda van Ekenstein $^{[12]}$, ou pela isomerização alcalina ${ }^{[5,21]}$. Portanto, elevados teores de lactulose indicam uso excessivo de temperatura no processo ${ }^{[12]}$. Em leite pasteurizado, a concentração de lactulose é de cerca de $0,3 \mathrm{~g} \mathrm{~L}^{-1[5,10,23]}$, observada no cromatograma da amostra, no tempo de retenção de 6,9 minutos, em baixa concentração em relação à lactose (Figura 4b). Já a ausência de degradação da lactose como exposto, embora com coeluição para a lactose e galactose, pode ser comprovada pelo pico da glicose que aparece no cromatograma da mistura de padrões injetada (Figura 4c), enquanto não é detectado na amostra (Figura 4b). Portanto, o aparecimento de picos adicionais indica forte indício de fraude, que se recomenda seja investigada.
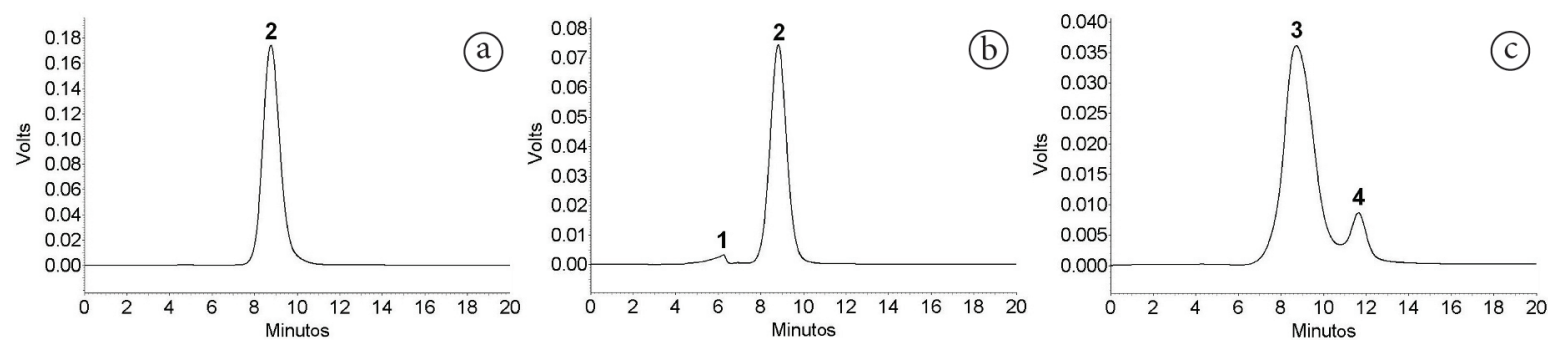

Figura 4 Cromatograma do padrão de lactose 5,0\% (m/v) (a); leite pasteurizado integral comercial com extração realizada utilizando leite: etanol (4: 1, v/v), em 10.000 rpm, por 37 minutos (b); padrão de lactose 1,5\%, galactose 0,25\% e glicose 0,25\% (m/v) (c); pico (1) lactulose, (2) lactose, (3) lactose e galactose, (4) glicose. 


\section{Conclusões}

O planejamento fatorial composto central propiciou encontrar as condições otimizadas de máxima extração da lactose para o leite pasteurizado, empregando o etanol como diluente de pré-tratamento, permitindo sua quantificação utilizando água como fase móvel no sistema cromatográfico.

O método desenvolvido e validado para determinação da lactose empregando cromatografia líquida com índice de refração mostrou-se adequado por ser preciso, linear em uma ampla faixa dinâmica, seletivo, específico e exato, com recuperação dentro dos limites aceitáveis.

\section{Agradecimentos}

Aos órgãos financiadores: CAPES, CNPq, Fundação Araucária e FINEP. Ao professor dr. Fábio Yamashita pelo auxílio com o planejamento experimental. Ao Laboratório Multiusuário LAPA da UEL, pelo uso da ultracentrífuga e sistema cromatográfico.

\section{Referências}

1 Siqueira KB, Zoccal R. Panorama do leite. Boletim eletrônico mensal 2013; 6(65).

2 Wattiaux MA. Composição do leite e seu valor nutricional. Instituto Babcock para Pesquisa e Desenvolvimento da Pecuária Leiteira Internacional; 2011. p. 74. Available from: http://babcock.wisc.edu/ sites/default/files/de/pt/de_19.pt.pdf. [cited 2013 May 12].

3 Silva PHF. Leite: Aspectos de composição e propriedades. Química Nova na Escola 1997; (6). [cited 2013 Oct 15]. Available from: http://qnesc.sbq. org.br/online/qnesc06/quimsoc.pdf.

4 Centro Integrado de Monitoramento da Qualidade dos Alimentos - CQuali. Cquali qualidade do leite - informações gerais. Cquali; 2008. [cited 2013 July 21]. Available from: http://www.cquali.gov.br/data/
Pages/MJ8F0048E8ITEMIDFBD8A1EB007A4CADB EF09F29C15C6431PTBRNN.htmi.

Kedzierska-Matysek M, Litwinczuk Z, Florek M, Barlowska J. The effects of breed and other factors on the composition and freezing point of cow's milk in Poland. International Journal of Dairy Technology 2011; 64(I.3):336-342.

6 Zhang LG, Zhang X, Ni LJ, Xue ZB, Gu X, Huang SX. Rapid identification of adulterated cow milk by non-linear pattern recognition methods based on near infrared spectroscopy. Food Chemistry 2014; 145:342-348. http://dx.doi.org/10.1016/j. foodchem.2013.08.064

7 Lenardão EJ, Freitag RA, Dabdoub MJ, Batista ACF, Silveira CC. "Green Chemistry" - Os 12 princípios da química verde e sua inserção nas atividades de ensino e pesquisa. Química Nova 2003; 26(1):123-129. http:// dx.doi.org/10.1590/S0100-40422003000100020

8 Dionex. Application Update 182. Measuring Lactose in Milk: A Validated Method. [cited 2013 Oct 15]. Available from: http://www.dionex.com/en-us/ webdocs/110944-AU182-HPLC-Lactose-Milk29Jul2011-LPN2894.pdf.

9 Analysis of lactose free dairy. Food Testing and Agriculture. [cited 2013 Nov 6]. Available from: http:// www.chem.agilent.com/Library/applications/59912252EN.pdf.

10 Determination of lactose in lactose-free milk products by high-performance anion-exchange chromatography with pulsed amperometric detection. Application note 248. [cited 2013 Nov 6]. Available from: http://www.dionex.com/en-us/ webdocs/87238-AN248-IC-Lactose-Milk-ProductsHPAE-PAD-05Oct2012-AN70236_E.pdf.

11 Xinmin W, Ruili Z, Zhihua L, Yuanhong W, Tingfu J. Determination of glucosamine and lactose in milk-based formulae by high-performance liquid chromatography. Journal of Food Composition and Analysis 2008; 21:255-258. http://dx.doi.org/10.1016/j. jfca.2007.10.006

12 Manzi P, Pizzoferrato L. HPLC determination of lactulose in heat treated milk. Food and Bioprocess Technology 2013; 6(3): 851-857. http://dx.doi. org/10.1007/s11947-011-0700-x

13 International Conference on Harmonisation - ICH. Q2(R1) Validation of analytical procedures: text and methodology. Geneva: ICH Secretariat; 2005. [cited 2013 Oct 15]. Available from: http://www. 
ich.org/fileadmin/Public_Web_Site/ICH_Products/ Guidelines/Quality/Q1A_R2/Step4/Q1A_R2_ Guideline.pdf.

14 Jenke DR. Chromatographic method validation II. Guidelines for primary validation parameters. Journal of Liquid Chromatography \& Related Technologies 1996; 19:737-757. http://dx.doi. org/10.1080/10826079608005534

15 Huber L. Good Laboratory Practice: a primer for high performance liquid chromatography, capillary electrophoresis and UV-visible spectroscopy. HewlettPackard; 1993.

16 Agência Nacional de Vigilância Sanitária - Anvisa. Resolução RE n 899, de 29 de maio de 2003. Guia para validação de métodos analíticos e bioanalíticos. Diário Oficial da República Federativa do Brasil, Braslília, 2 jun. 2003. Seção 1.

17 Jogleka AM, May AT. Product excellence through design of experiments. Cereal Foods World 1987; 32(12):857-868.

18 Brito, A. B. N. Estudo da cristalização da lactose em diferentes solventes. 2007. 132p. Tese (Doutorado) Universidade Federal de São Carlos, São Paulo.
19 Embrapa. Agência de Informação Embrapa. $p H$ do Leite. [cited 2013 Nov 8]. Available from: http://www. agencia.cnptia.embrapa.br/Agencia8/AG01/arvore/ AG01_193_21720039246.html.

20 Dharmasetiawani N, Suryadi H, Firmansyah A. Quantitative analysis of lactose and lactulose in urine by high performance liquid chromatography for determination of intestinal lactase activity. Medical Journal of Indonesia 2003; 12:8-12. http://dx.doi. org/10.13181/mji.v12i1.84

21 Aider M, Halleux D. Isomerization of lactose and lactulose production: review. Trends in Food Science \& Technology 2007; 18:356-364. http://dx.doi. org/10.1016/j.tifs.2007.03.005

22 Fresenius-Kabi. Lactulose - From milk sugar to lactulose. [cited 2013 Nov 5]. Available from: http:// www.freseniuskabi-productpartnering.com/4263. htm.

23 Fattori DC. Galactosilação por processo térmico do soro do leite da produção de queijo. [cited 2013 Nov 8]. Available from: http://webcache.googleusercontent. com/search?q=cache: 1 UsKYl0M0ukJ:www.prppg. ufpr.br/sites/default/files/webform/posterevinci20/ siepe_modelo_banner_1_1_8.ppt+\&cd =1\&hl=pt$\mathrm{BR} \& \mathrm{ct}=\mathrm{clnk} \& \mathrm{gl}=\mathrm{br}$. 